\title{
Model of Temperature Dependence of Spontaneous Polarization of Thiourea Monocrystal in Phase I
}

\author{
I. ŚliWa AND W. WANARSKI \\ Institute of Molecular Physics, Polish Academy of Sciences \\ M. Smoluchowskiego 17, PL 60-179 Poznań, Poland
}

(Received November 15, 2011; in final form February 8, 2012)

\begin{abstract}
In this paper we present a model of spontaneous polarization appearing in the crystal of thiourea in phase I, constructed on the basis of the molecular field theory, with the use of the Boltzmann statistics. The model employs the potential energy of interaction between two nonpunctual molecular electric dipoles performing reorientations within the constraints specified by the experimentally determined structural data. The temperature dependences of the spontaneous polarization for different values of the hydrostatic pressure and for different $\gamma$-ray doses were obtained. A comparison between theoretical and experimental results permitted calculation of the parameters of the theory presented.
\end{abstract}

PACS: 77.80.Bh, 77.80.-e

\section{Introduction}

On the basis of the results presented in paper [1], the energy of interaction of a pair of substitute nonpunctual molecular electric dipoles of thiourea monocrystal can be described by the following formula:

$$
U=-2 E_{0} m\left(\sqrt{\delta^{2}-\cos ^{2} x}-s \sin ^{2} x-\sigma \sin x\right),
$$

with $x=\theta / 2, s=2 a m / E_{0}, \sigma=b P \cos \beta / E_{0}, \delta=L / d$, where $\theta$ is the angle of reorientation of a given pair of dipoles, $a$ is a short-range interaction coefficient, $b$ is a long-range interaction coefficient, $P$ is the polarization of the monocrystal of thiourea, $E_{0}$ denotes the value of intensity of the local electric field acting on the nonpunctual molecular dipoles, $m$ is the value of the electric dipole moment of the molecule of thiourea, $d$ is the length of the nonpunctual molecular electric dipole of thiourea; $\beta$ denotes the angle between the line perpendicular to the bisectrix of the angle of reorientation and the ferroelectric axis, and $L$ is the length of the segment describing the restricted possibilities of reorientation of a given pair of dipoles (Fig. 1).

Depending on the value of the length $L$, the diagram of the potential energy $U$ as a function of the angle of reorientation $x$ can take different forms. The quantity $L$ is determined by the lattice constants $a, b, c$ which are temperature-dependent. The temperature dependence of the lattice constants was studied in the article of Futama [2]. In the range of the ferroelectric phase transitions it reveals some anomalies.

\section{The model}

From the results of the structural studies of thiourea monocrystal, reported in articles [3] and [4], and on the basis of formula (1), the relative potential energy of inter-

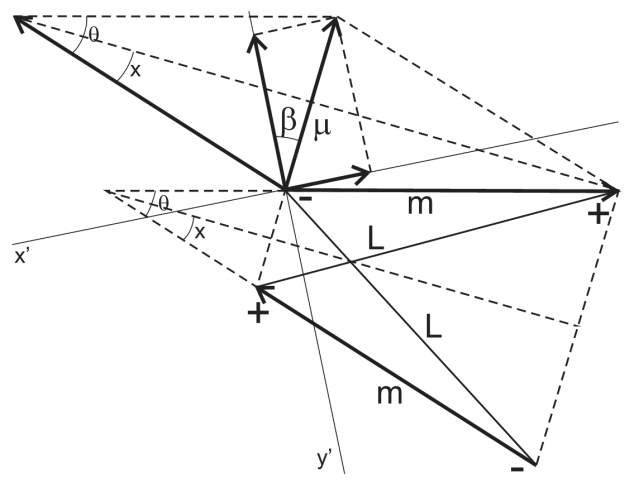

Fig. 1. Projection of the pair of the substitute nonpunctual molecular electric dipoles in the crystal of thiourea onto the $x^{\prime} y^{\prime}$ plane, with restricted possibilities of reorientation ( $L=$ const) and the resultant dipole moment $\mu$.

action $U_{w}=U / 2 m E_{0}$ of a pair of the substitute nonpunctual molecular electric dipoles in the absence of external electric field can be depicted as a curve symmetric to the angle $x$ of reorientation of the dipoles (Fig. 2 and Fig. 3).

The diagrams presented in Figs. 2 and 3 correspond to the paraelectric and antiferroelectric states, respectively. For non-zero polarization of thiourea monocrystal $(\sigma \neq$ 0 ), on the basis of formula (1), the diagrams in Figs. 2 and 3 are transformed into the diagrams presented in Fig. 4 and Fig. 5.

If the crystal is polarized, the potential energy depicted in Fig. 3 changes, as illustrated in Fig. 5. The two potential minima are not equivalent. Analyzing the function $U(x)$ given by formula (1), in the linear approximation $(\sigma \ll 1)$ we obtain the formulae for the sinuses of the angles $\left(x_{1}\right.$ and $\left.x_{2}\right)$ corresponding to minima and the sinus 


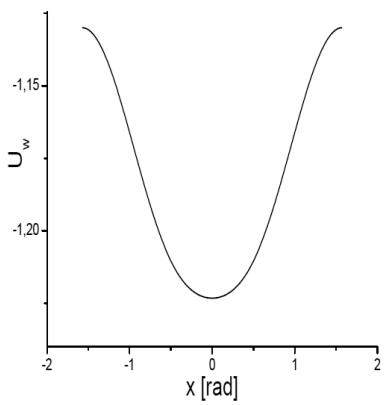

Fig. 2. The energy of interaction of the pair of substitute nonpunctual molecular electric dipoles of thiourea monocrystal as a function of the angle of reorientation $x$, for zero polarization of the crystal $(\sigma=0)$ and for $\delta^{2}>1+1 / 4 s^{2}([1]$, Fig. 8 b) .

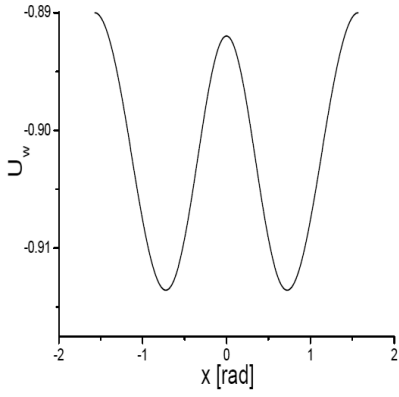

Fig. 3. The energy of interaction of the pair of substitute nonpunctual molecular electric dipoles of thiourea monocrystal as a function of the angle of reorientation $x$, for zero polarization of the crystal $(\sigma=0)$, and for $1 / 4 s^{2}<\delta^{2}<1+1 / 4 s^{2}$ ([1], Fig. 8a).

of the angle $\left(x_{3}\right)$ corresponding to the maximum

$$
\begin{aligned}
& \sin x_{1}=\sin x_{0}-\sigma /\left(y_{0}^{2}(2 s)^{3}\right), \\
& \sin x_{2}=-\sin x_{0}-\sigma /\left(y_{0}^{2}(2 s)^{3}\right), \\
& \sin x_{3}=\sqrt{\delta^{2}-1} \sigma /\left(1-2 s \sqrt{\delta^{2}-1}\right),
\end{aligned}
$$

where

$$
y_{0}=\sin x_{0}=\sqrt{1+1 / 4 s^{2}-\delta^{2}}
$$

is the modulus of the sinus of the angle corresponding to minimum of the function $U_{w}(x)$, for $\sigma=0$ and $1 / 4 s^{2}<\delta^{2}<1+1 / 4 s^{2}$.

In the linear approximation, the difference between the energy $U(x)$ for the angles corresponding to minima of this function, is given by

$$
U_{w}\left(x_{1}\right)-U_{w}\left(x_{2}\right)=4 E_{0} m y_{0} \sigma .
$$

Denoting: $U_{w}\left(x_{1}\right)-U_{w}\left(x_{2}\right)=2 \Delta$, we obtain the formula for the quantity $\Delta$ being a measure of the difference between the minima of the potential energy

$$
\Delta=2 E_{0} m y_{0} \sigma .
$$

From Fig. 1 and on the basis of (2), (3) and (5) it appears that the projections of the substitute electric dipole moments of a given pair of dipoles onto the ferroelectric axis $y$ can take the values determined by the formulae

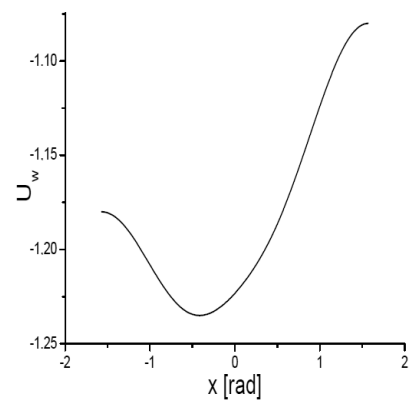

Fig. 4. The energy of interaction of the pair of substitute nonpunctual molecular electric dipoles of thiourea monocrystal as a function of the angle of reorientation $x$, for non-zero polarization of the crystal $(\sigma \neq 0)$, if $\delta^{2}>1+\left(1-\sigma^{2 / 3}\right)^{3} / 4 s^{2}$ ([1], Fig. 9c).

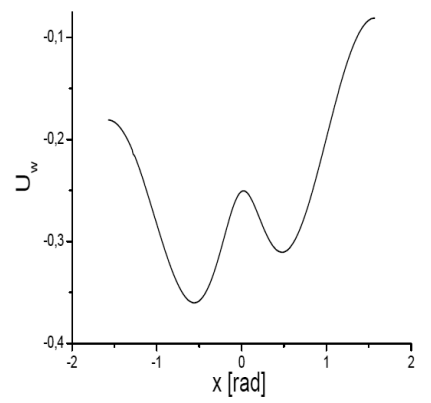

Fig. 5. The energy of interaction of the pair of substitute nonpunctual molecular electric dipoles of thiourea monocrystal as a function of the angle of reorientation $x$, for non-zero polarization of the crystal $(\sigma \neq 0)$, if $1 /(2 s-\sigma)^{2}<\delta^{2}<1+\left(1-\sigma^{2 / 3}\right)^{3} / 4 s^{2}$ ([1], Fig. 9a).

$$
\begin{aligned}
& \mu_{1}=2 m\left[y_{0}-\sigma /\left(y_{0}^{2}(2 s)^{3}\right)\right] \cos \beta, \\
& \mu_{2}=2 m\left[-y_{0}-\sigma /\left(y_{0}^{2}(2 s)^{3}\right)\right] \cos \beta .
\end{aligned}
$$

Assuming that in the crystal there are $N_{1}$ pairs of dipoles oriented at angle $\theta_{1}=2 x_{1}$ and $N_{2}$ pairs of dipoles oriented at angle $\theta_{2}=2 x_{2}$, the polarization of the crystal can be expressed as follows:

$$
P=N_{2} \mu_{2}-N_{1} \mu_{1} \text {, }
$$

and the total number of the pairs of dipoles equals

$$
N=N_{1}+N_{2} \text {. }
$$

From (10) and (11) it appears that the numbers $N_{1}$ and $N_{2}$ are equal to

$$
\begin{aligned}
& N_{1}=\left(N \mu_{2}-P\right) /\left(\mu_{1}+\mu_{2}\right), \\
& N_{2}=\left(N \mu_{1}+P\right) /\left(\mu_{1}+\mu_{2}\right) .
\end{aligned}
$$

In the conditions of thermodynamic equilibrium with environment, the polarization of the crystal depends on its temperature and, according to the Boltzmann statistics, it can be described by the formula (Fig. 5):

$$
P(T)=N_{2} \mu_{2} p_{2}-N_{1} \mu_{1} p_{1}
$$

where $p_{1}$ and $p_{2}$ are the probabilities that a given pair of dipoles is in the state 1 or 2 , determined as follows: 


$$
p_{1}=\frac{1}{Z} \mathrm{e}^{-\Delta / k T}, \quad p_{2}=\frac{1}{Z} \mathrm{e}^{\Delta / k T},
$$

where $k$ denotes the Boltzmann constant, and $Z$ is the sum-of-states of a given pair of dipoles, which has the form

$$
Z=\mathrm{e}^{-\Delta / k T}+\mathrm{e}^{\Delta / k T} .
$$

The quantity $\Delta$, on the basis of formulae (1) and (7), is equal to

$$
\Delta=2 m y_{0} b P \cos \beta \text {. }
$$

Substituting (15), (16), (17), (13), (12), (8) and (9) into formula (14) after simple transformations we obtain the equation for the temperature dependence of the relative polarization of the crystal $P_{w}(T)$ :

$$
C P_{w}^{2} /\left(y_{0}^{2}-C^{2} P_{w}^{2}+y_{0} P_{w}\right)=\tanh \left(D P_{w} / y_{0}\right)
$$

where

$$
\begin{aligned}
& P_{w}=P / P_{N}, \quad P_{N}=2 N m \cos \beta, \\
& C=b P_{N} \cos \beta /\left((2 s)^{3} y_{0}^{2} E_{0}\right), \\
& D=b y_{0}^{2} P_{N}^{2} /(N k T) .
\end{aligned}
$$

According to the structural data from [4] $\delta=1.073$ for phase $\mathrm{V}$ and $\delta=1.031$ for phase I of the crystal of thiourea. Within the approximation $\delta \approx 1$, from (5) we obtain

$$
y_{0} \approx 1 / 2 s \text {. }
$$

Applying the Onsager molecular field theory, according to the suggestion given in [1], we could assume

$$
a=b / V \text { and } P \cos \beta=M / V,
$$

where $V$ is the volume of the Onsager alcove containing a pair of interacting nonpunctual molecular electric dipoles in the crystal of thiourea, and $M$ denotes the electric dipole moment of that homogeneously polarized alcove. Substituting the formulae (23) into (20) and (21) we obtain

$$
C=\frac{1}{2} N V \cos ^{2} \beta
$$

and

$$
D=C E_{0}^{2} /(2 a k T)
$$

or

$$
D=C G,
$$

where

$$
G=E_{0}^{2} /(2 a k T) .
$$

From the above expressions it appears that $C$ is crystal volume dependent and $G$ is inversely proportional to its temperature $T$. Equation (18) can be solved numerically for different values of $C$ and $y_{0}$. As a result, we obtain the temperature dependence of relative polarization $P_{w}$. Exemplary solutions are presented in Fig. 6 .

The diagram presented in Fig. 6 consists of three branches. Branches 2 and 3 must be rejected because they imply nonphysical effects such as increase in the modulus of polarization with increasing temperature and polarization existence for any finite temperature, which are difficult to explain. Figure 7 illustrates the temper-

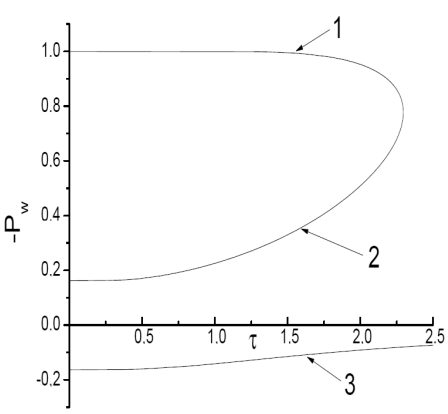

Fig. 6. The relative polarization $P_{w}$ of thiourea crystal as a function of the relative temperature $T=G^{-1}$.

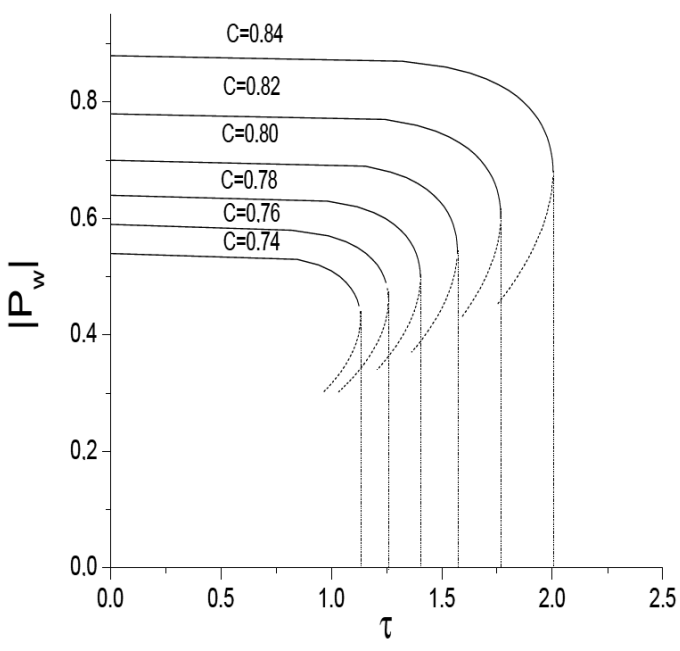

Fig. 7. The modulus of the relative spontaneous polarization of thiourea crystal as a function of temperature, for different values of parameter $C$ and for a constant value of $y_{0}=0.14$ (fragments of the rejected branch 2 are depicted with the dashed line).

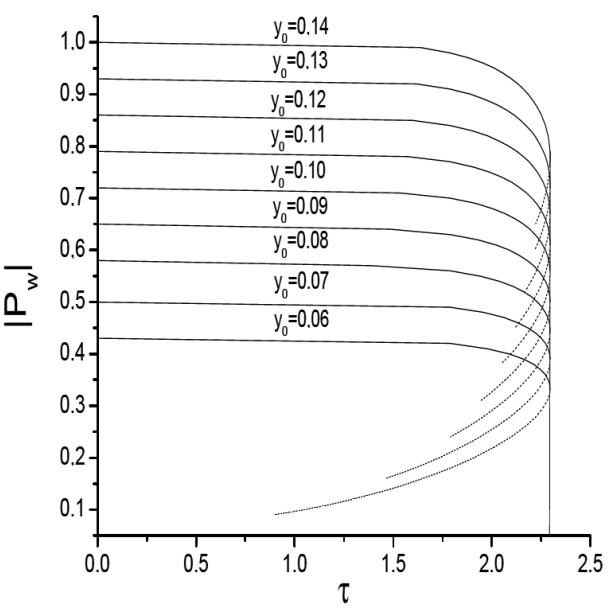

Fig. 8. The modulus of the relative spontaneous polarization of thiourea crystal as a function of temperature, for different values of $y_{0}$ and for a constant value of parameter $C=0.86$ (fragments of the rejected branches 2 are depicted with the dashed line). 
ature dependence of relative spontaneous polarization, taking into account branch 1 , for different values of parameter $C$ and for a constant value of $y_{0}=0.14$. The temperature dependence of relative spontaneous polarization for different values of $y_{0}$ and for a constant value of parameter $C=0.86$ is presented in Fig. 8 .

From Figs. 7 and 8 it follows that spontaneous polarization appears abruptly at the Curie point, and in low temperatures it very weakly depends on the temperature changes. In Fig. 7 we can see that with decreasing $C$ the spontaneous polarization decreases and the Curie point is shifted toward lower temperatures. According to Fig. 8 the spontaneous polarization decreases with decreasing value of the parameter $y_{0}$, but the Curie temperature is preserved.

\section{Discussion}

The obtained theoretical temperature dependence of the relative spontaneous polarization of thiourea monocrystal, shown in Fig. 8 and Fig. 9, can be compared to the experimental results presented in articles [5] and [6] (Figs. 9 and 10).

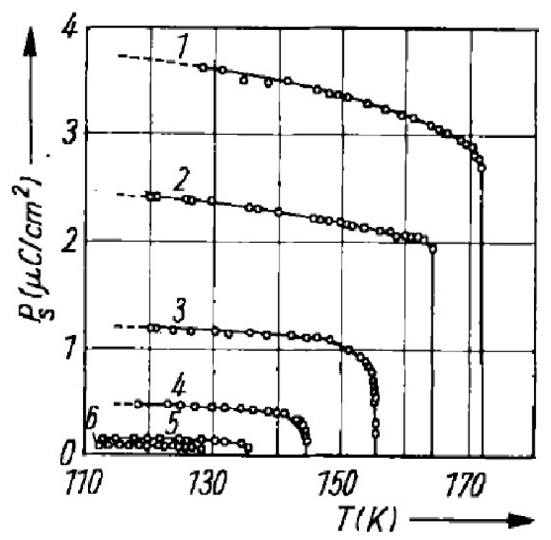

Fig. 9. The temperature dependence of spontaneous polarization of the monocrystal of thiourea in phase I, for different values of hydrostatic pressure: 1 - atmospheric pressure, $2-330$ bar, $3-600$ bar, $4-970$ bar, $5-1210$ bar, $6-1490$ bar [5].

Analyzing Figs. 7-10, we can notice the affinity between Figs. 7 and 9 and the affinity between Figs. 8 and 10 , respectively.

From the formula (24) it follows that parameter $C$ is directly proportional to the number $N$ of dipole pairs considered and to the volume $V$ of the Onsager alcove, which means that it is directly proportional to the volume of the crystal. Therefore, the effect of hydrostatic pressure on the spontaneous polarization of the monocrystal of thiourea in phase I can be explained classically - as a result of the crystals volume decrease upon increasing pressure. Similarly, on the basis of formulae (1) and (22) it is evident that a decrease in the value of $y_{0}$ is caused by decreasing value of the expression $E_{0} /(\mathrm{am})$, so it can

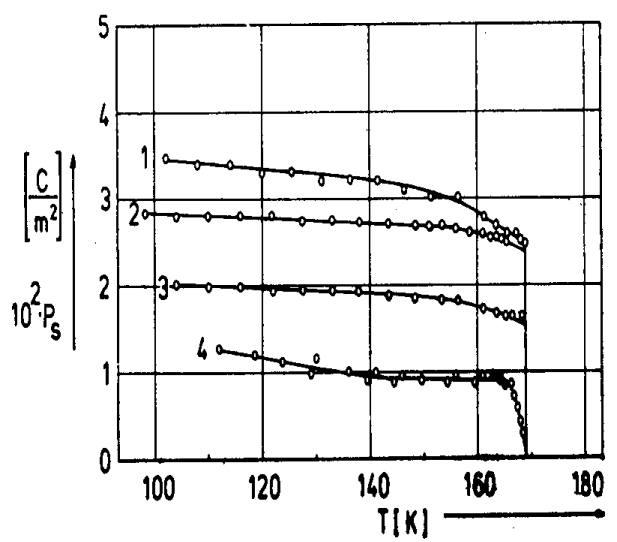

Fig. 10. The temperature dependence of spontaneous polarization of the monocrystal of thiourea in phase I for different $\gamma$-ray doses: $1-0 \mathrm{Mrad}, 2-22.1 \mathrm{Mrad}$, 3 - 41.6 Mrad, 4 - 100 Mrad [6].

be connected with the change in the intermolecular interactions and with the change in the value of electric dipole moment of the molecule, which can lead to the appearance of defects in the crystal, as it has also been found in article [7]. According to the presented theory, the value of the spontaneous polarization at the Curie point, for a constant value of coefficient $C$, decreases linearly with decreasing value of parameter $y_{0}$, which is illustrated in Fig. 11.

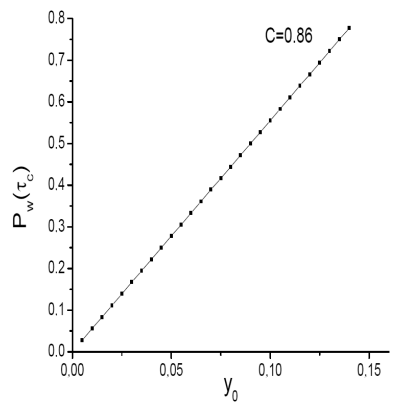

Fig. 11. The relative spontaneous polarization of thiourea crystal at the Curie point as a function of the parameter $y_{0}$, for a constant value of $C=0.86$.

A similar dependence of the relative spontaneous polarization of thiourea crystal in phase I on $y_{0}$ at constant temperature was obtained experimentally in [6]. The experiment was performed with increasing doses of $\gamma$-radiation, which is an additional argument to supporting that $\gamma$-radiation influences the change in the intermolecular interaction and the change in the value of electric dipole moment of the molecule (so it is described by the change in parameter $y_{0}$ ).

Assuming that the value of the crystal volume $V_{k}$ depends on the hydrostatic pressure $p$ according to the formula 


$$
V_{k}=V_{0}(1-\kappa p),
$$

where $\kappa$ is the compressibility factor, and $V_{0}=V_{k}(p=0)$, on the basis of (24) we can connect the coefficient $C$ with the volume of the crystal $V_{k}$ in the following way: $C=A V_{k}$, where $A=0.5 \cos ^{2} \beta, V_{k}=N V$. So, from (28) it follows that:

$$
C=C_{0}(1-\kappa p),
$$

where $C_{0}=A V_{0}$. Next, from (29) we obtain

$$
\kappa p=\left(C_{0}-C\right) / C_{0},
$$

so, if the values of the coefficients $C$ and $C_{0}$ are known, we can determine the value of the hydrostatic pressure, accurate to a multiplicative constant. On the basis of Eq. (18), taking into account the formulae (26), (27) and (30), the dependence of the relative Curie temperature $\tau_{\mathrm{c}}=2 a k T / E_{0}^{2}$ on $\kappa p$ was plotted (Fig. 12).

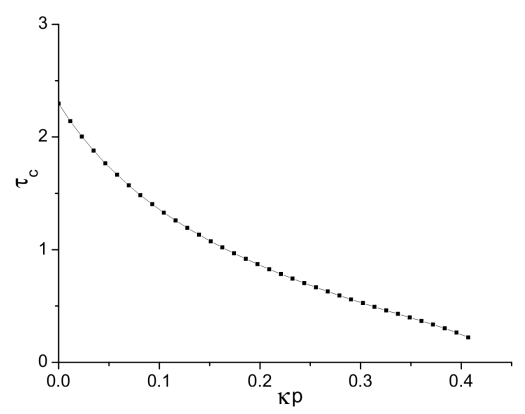

Fig. 12. The relative Curie temperature dependence $\tau_{\mathrm{c}}$ on $\kappa p$.

Comparing Fig. 12 with the pressure dependence of the Curie temperature from [5] $\left(\mathrm{d} T_{\mathrm{c}} / \mathrm{d} p=35.35 \times\right.$ $10^{-8} \mathrm{deg} / \mathrm{Pa}$ ), we obtain the value of the compressibility factor of thiourea crystal as $\kappa=4.02 \times 10^{-10} \mathrm{~Pa}^{-1}$. The use of the above formulae makes it easy to obtain the following values of the parameters of the presented theory:

- the value of the Onsager alcove $(p=0) V=3.4 \times$ $10^{-28} \mathrm{~m}^{3}$

- the radius of the Onsager alcove $(p=0) r=4.3 \times$ $10^{-10} \mathrm{~m}$,

- the short-range interaction coefficient $a=2.4 \times$ $10^{37} \mathrm{~V} /\left(\mathrm{C} \mathrm{m}^{2}\right)$,

- the long-range interaction coefficient $b=8.4 \times$ $10^{9} \mathrm{~V} \mathrm{~m} / \mathrm{C}$,

- the intensity of the local electric field $E_{0}=2.2 \times$ $10^{8} \mathrm{~V} / \mathrm{m}$,

being in conformity with the structure of the crystal of thiourea (for example the radius and the value of the Onsager alcove).

The compressibility factor obtained was found to be of the same order as for other molecular crystals [8].
According to [9], in the calculations we put the electric dipole moment of thiourea molecule in the crystal as $m=$ $4.86 \mathrm{D}$.

The theoretical model presented in this paper is based on the electrostatic interactions between the molecules of the crystal of thiourea. Such approach is in conformity with the conclusions given in [10], where the great role of the electrostatic and polarization effects on the intermolecular interactions in linear arrays of urea, thiourea and their derivatives (with varying chain length) was emphasized. Such approach emphasizes the electrostatic nature of the dipole-dipole interactions in these systems, which is in conformity with the model presented in this paper.

\section{Conclusions}

In this paper and in paper [1] the assumption that the dipoles of molecules are of finite length and that they can perform reorientations only within certain constraints, led to the unconventional form of the potential energy of interaction between these dipoles in the crystal as a function of the angle between them (such an energy dependence has one or two minima).

The difference in values between two inequivalent minima of the potential energy of interaction of two nonpunctual molecular electric dipoles in thiourea crystal is in linear approximation directly proportional to the polarization of the crystal.

Applying the obtained potential energy of interaction with two inequivalent minima to the problem of $N$ interacting pairs of nonpunctual molecular electric dipoles, within the Boltzmann statistics and in the molecular field approximation, the dependence of the relative spontaneous polarization on the relative temperature was obtained.

A qualitative agreement between the theoretical and experimental temperature dependence of the spontaneous polarization of thiourea crystal in phase I was found: for the non-irradiated crystal under different hydrostatic pressure and for the crystal under the atmospheric pressure, irradiated with different doses of $\gamma$-radiation.

Within the theory presented in this paper it was found that the changes in the temperature dependence of spontaneous polarization of thiourea in phase I were caused: by the classical change in the volume of the crystal under varying hydrostatic pressure and by the change in the intermolecular interactions and in the dipole moment of the molecule upon $\gamma$-radiation, respectively.

From comparison of the theoretical results with the experimental ones, the compressibility factor of thiourea, as well as the other parameters of the theory presented (for example, the volume and the radius of the Onsager alcove) were derived. 


\section{References}

[1] I. Śliwa, W. Wanarski, Acta Phys. Pol. A 120, 516 (2011).

[2] H. Futama, J. Phys. Soc. Jpn. 17, 434 (1962).

[3] G.J. Goldsmith, J.G. White, J. Chem. Phys. 31, 1175 (1959).

[4] M.M. Elcombe, J.C. Taylor, Acta Crystallogr. A 24, 410 (1968).

[5] J. Klimowski, W. Wanarski, D. Ożgo, Phys. Status Solidi A 34, 697 (1976).
[6] W. Wanarski, Acta Phys. Pol. A 56, 197 (1979).

[7] A. Pawłowski, The Physics of Dielectrics and Radiospectroscopy, Vol. IX/2, PTPN, Warszawa 1977 , p. 223.

[8] A.I. Kitaygorodsky, Molecular Crystals, PWN, Warszawa 1976

[9] C. Calvo, J. Chem. Phys. 33, 1721 (1960).

[10] S. Pal, A.K. Manna, S.K. Pati, J. Chem. Phys. 129 204301 (2008). 